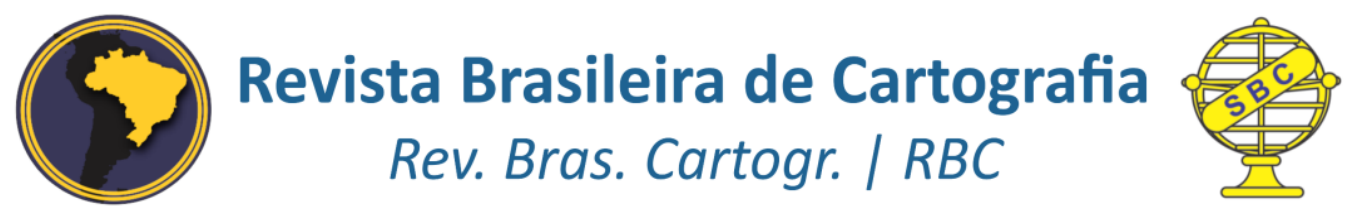

DOI: http://dx.doi.org/10.14393/rbcv71n3-46316

\title{
Desenvolvimento e Perspectivas da Infraestrutura de Dados Espaciais Marinhos Brasileira
}

\author{
Development and Perspectives of Brazilian Marine Spatial Data \\ Infrastructure
}

Christopher Florentino ${ }^{1}$

Vitor Bravo Pimentel ${ }^{2}$

Arthur Ayres Neto ${ }^{3}$

Recebido em dezembro de 2018.

Aprovado em junho de 2019.

\begin{abstract}
RESUMO
O conceito de Infraestrutura de Dados Espaciais Marinhos (IDEM) tem conquistado espaço ao redor do globo representando o desenvolvimento da dimensão marinha em uma Infraestrutura de Dados Espaciais (IDE). Consiste basicamente, num conjunto de ações coordenadas envolvendo tecnologias, padrões, dados espaciais e pessoas interessadas na gerência e uso compartilhado de recursos e informações em prol do bem comum. Estas seguem apoiadas por diretivas nacionais e internacionais, em destaque aquelas emanadas no Brasil, pelo Plano de Ação para Implantação da INDE (CONCAR, 2010) e pela Norma de Acesso aos Dados e às Informações Abertas da Diretoria de Hidrografia e Navegação (DHN, 2018), bem como as orientações advindas da Organização Hidrográfica Internacional (OHI). As IDEM's têm se mostrado uma eficiente ferramenta de gerenciamento das atividades humanas no tocante a utilização racional e de disponibilidade duradoura dos recursos ambientais, integrando assim iniciativas com vistas ao chamado "uso inteligente dos oceanos". Assim, com base numa revisão bibliográfica e no uso de alguns exemplos, apresenta-se o relacionamento harmônico entre IDE's e IDEM's em escalas nacionais e regionais, destacando alguns dos potenciais benefícios e desafios de sua implantação e disponibilização para a comunidade marítima brasileira.
\end{abstract}

PALAVRAS-CHAVE: Infraestrutura de Dados Espaciais Marinhos (IDEM). Infraestrutura Nacional de Dados Espaciais (INDE). batimetria.

\footnotetext{
${ }^{1}$ Diretoria de Hidrografia e Navegação (DHN) / Marinha do Brasil (MB) e Programa de Pós-graduação em Dinâmica dos Oceanos e da Terra da Universidade Federal Fluminense (UFF), Brasil. E-mail: christopherf@id.uff.br.

${ }^{2}$ Diretoria de Hidrografia e Navegação (DHN) / Marinha do Brasil (MB) e Programa de Pós-graduação em Dinâmica dos Oceanos e da Terra da Universidade Federal Fluminense (UFF), Brasil. E-mail: vbpimentel@id.uff.br.

${ }^{3}$ Departamento de Geociências. Programa de Pós-graduação em Dinâmica dos Oceanos e da Terra da Universidade Federal Fluminense (UFF), Brasil. E-mail: aayres@id.uff.br.
} 


\begin{abstract}
The concept of Marine Spatial Data Infrastructures (MSDI) has achieved worldwide coverage representing the development of marine dimension of Spatial Data Infrastructure (SDI). It basically consists of a framework which coordinates actions involving technologies, standards, spatial data and people responsible for managing and sharing resources and information focusing on common goods. The MSDI is supported by national and international guiding principles and rules, as those promulgated in Brazil, as the Action Plan for the Implementation of the NSDI (CONCAR, 2010) and the Access Policy to Open Data and Information of Directorate of Hydrography and Navigation (DHN, 2018), as well as those guidelines from the International Hydrographic Organization (IHO). The MSDI initiatives are an efficient tool for managing human activities regarding the rational uses and sustainable availability of natural resources, merging those approaches towards the smart use of oceans. Therefore, through a theoretical review and some cases studies, this paper presents an analysis of harmonic relation between SDI and MSDI in a national and regional context, highlighting some of potential benefits and challenges of its implementation for the Brazilian maritime community.
\end{abstract}

KEYWORDS: Marine Spatial Data Infrastructure (MSDI). National Spatial Data Infrastructure (NSDI). bathymetry.

\title{
Introdução
}

Representando uma tendência mundial para a manutenção da vida por meio da gestão estratégica dos recursos naturais dos mares e oceanos, as Infraestruturas de Dados Espaciais Marinhos (IDEM's) seguem em franco desenvolvimento por diversos países (IHO, 2019a). Casos como da Alemanha (SEIP e BILL, 2016; MDI-DE, 2018), Dinamarca (HARTMANN, 2015; IHO, 2019b), Canadá (NRCAN, 2018), Croácia (TAVRA, LEDER e CETL, 2018), Malásia (MyGDI, 2018), Singapura (CHEN, 2019) e Brasil (DHN, 2016; 2018), demonstram a pujança das IDEM's que se mostram em diferentes níveis de maturidade de implementação ou operação. Tais iniciativas são apoiadas pela Organização Hidrográfica Internacional (OHI) (MARATOS, 2006; IHO, 2017a; 2017b) em conjunto de outras instituições e projetos governamentais (UN-GGIM WGMGI, 2019; INSPIRE, 2018) e nãogovernamentais (IHO, 2019a; SMART OCEANS, 2018). Alguns dos benefícios do uso de IDEM incluem desde o estabelecimento de um tráfego marítimo 
seguro e eficiente, o aperfeiçoamento na gestão da zona costeira, a exploração sustentável e a otimização de recursos marinhos, a proteção ambiental e a defesa nacional adequadas (COOPER, PEPPER e OSBORNE, 2010; CONTI et al., 2013).

Entretanto, a indisponibilidade de acesso aos dados e informações espaciais de forma simples e ágil, por vezes tem comprometido avanços nacionais em diversas áreas, tal como o caso das pesquisas científicas, impossibilitando que os pesquisadores dediquem maior tempo de análise sobre os elementos espaciais e suas interações com o ambiente. No Brasil, tal situação foi apontada durante o workshop internacional Marine Data Management, na sede da Fundação de Amparo à Pesquisa do Estado de São Paulo (FAPESP), que destacou a necessidade da busca por soluções geoespaciais integradas (FAPESP, 2012).

Desta forma, o presente artigo tem como objetivo geral apresentar uma análise sobre a evolução do emprego das Infraestruturas de Dados Espaciais (IDE's) sobre os domínios continental e marinho nos últimos anos. Ademais, adotando como prisma específico ao tema, intenciona-se discorrer sobre o desenvolvimento do atual projeto de implementação da IDEM brasileira. Para tal, a seção 2 trará uma breve revisão dos conceitos pertencentes às IDE's, discorrendo a respeito de algumas iniciativas adotadas ao redor do globo para construção de Infraestruturas Nacionais de Dados Espaciais (INDE's) e das Infraestruturas de Dados Espaciais Marinhos (IDEM's). A seção 3 apresentará o relacionamento harmônico entre as IDE's e IDEM's e suas principais motivações, destacando os avanços nacionais. Logo, a seção 4 irá aprofundar sua análise discorrendo, à título de exemplo, sobre um tipo de dado espacial marinho, a batimetria. Assim, será possível verificar a abordagem deste conjunto de dados dentro do cenário internacional, bem como sua tendência mundial e perspectiva nacional.

Espera-se, por fim, contribuir para discussão sobre o intercâmbio de dados espaciais terrestres e marinhos, com base no uso de boas práticas à gestão de soluções tecnológicas sustentáveis. 


\section{Fundamentação Teórica}

\subsection{Principais Conceitos}

A ideia de uma Infraestrutura de Dados Espaciais (IDE) parte da organização de dados geoespaciais mediante o uso de tecnologias, padrões, políticas e acordos institucionais a fim de facilitar a geração, armazenamento, distribuição e o acesso destes ao público (MCLAUGHLIN, 1991; CROMPVOETS et al., 2004; BORBA et al., 2015; OGC, 2018). Para efeito da Infraestrutura Nacional de Dados Espaciais brasileira (INDE-BR), as entidades responsáveis por essa iniciativa seriam os Centro produtores tais como, as instituições do governo, a academia, autarquias públicas ou privadas e demais setores responsáveis pela produção e manutenção do respectivo dado ou informação geoespacial de referência, temáticas ou de valor agregado (BRASIL, 2008). Assim, tem-se que uma IDE visa otimizar recursos e potencializar os resultados entre os entes que dela participam ou usufruem. Por sua vez, a Infraestrutura de Dados Espaciais Marinhos (IDEM) segue este mesmo preceito, atribuindo destaque ao propósito marítimo relacionando seus respectivos conjuntos de dados em temas ou camadas de interesse à futuras análises (IHO, 2017a).

\subsection{O surgimento das Infraestruturas Nacionais de Dados Espaciais}

De acordo com Crompvoets et al. (2004) é possível notar a existência de estudos envolvendo o conceito de IDE, ou Spatial Data Infrastructures (SDI), em meados dos anos 80, com os exemplos dos Estados Unidos da América e da Austrália. Ainda que limitados pela própria inexperiência, cada país buscava soluções geográficas que integrassem o atendimento das demandas e prioridades específicas à suas características nacionais, dando início ao que hoje chamamos de National Spatial Data Infrastructure (NSDI) (FGDC, 1997). Contudo, após um amadurecimento impulsionado principalmente pelo 
desenvolvimento tecnológico e econômico, tem-se o surgimento de uma segunda geração de IDE's nos anos 2000. Ocorreu neste período uma mudança na forma de gestão do assunto, trazendo o uso das IDE's como uma boa prática voltada aos modelos de gerenciamento público e privado, permitindo analisar seus impactos no desenvolvimento sustentável das sociedades (RAJABIFARD, 2008). O trato das IDE's passou a seguir uma organização sistemática mais formal, sendo destacada a importância do uso de padrões para o armazenamento dos dados e seus metadados, da tecnologia envolvida e das políticas de gestão de todo esse conjunto (AALDERS e MOELLERING, 2001). Conforme apresenta a Figura 1, a distribuição das IDE's passa também por uma hierarquia em níveis de abrangência (local, nacional, regional, global...) e de sua estrutura organizacional (operacional, gerencial e estratégico). Houve ainda a criação de fóruns e comunidades voltadas a discussão do tema (RAJABIFARD, FEENEY e WILLIAMSON, 2002), como por exemplo o Open GIS Consortium Inc. em 1994, passando a Open Geospatial Consortium - OGC em 2004 (OGC, 2018), e do European Umbrella Organisation for Geographic Information - EUROGI de 1994 (EUROGI, 2018), que segue ativo junto da iniciativa INSPIRE (2018) desde 2007.

Figura 1 - A organização hierárquica das IDE's

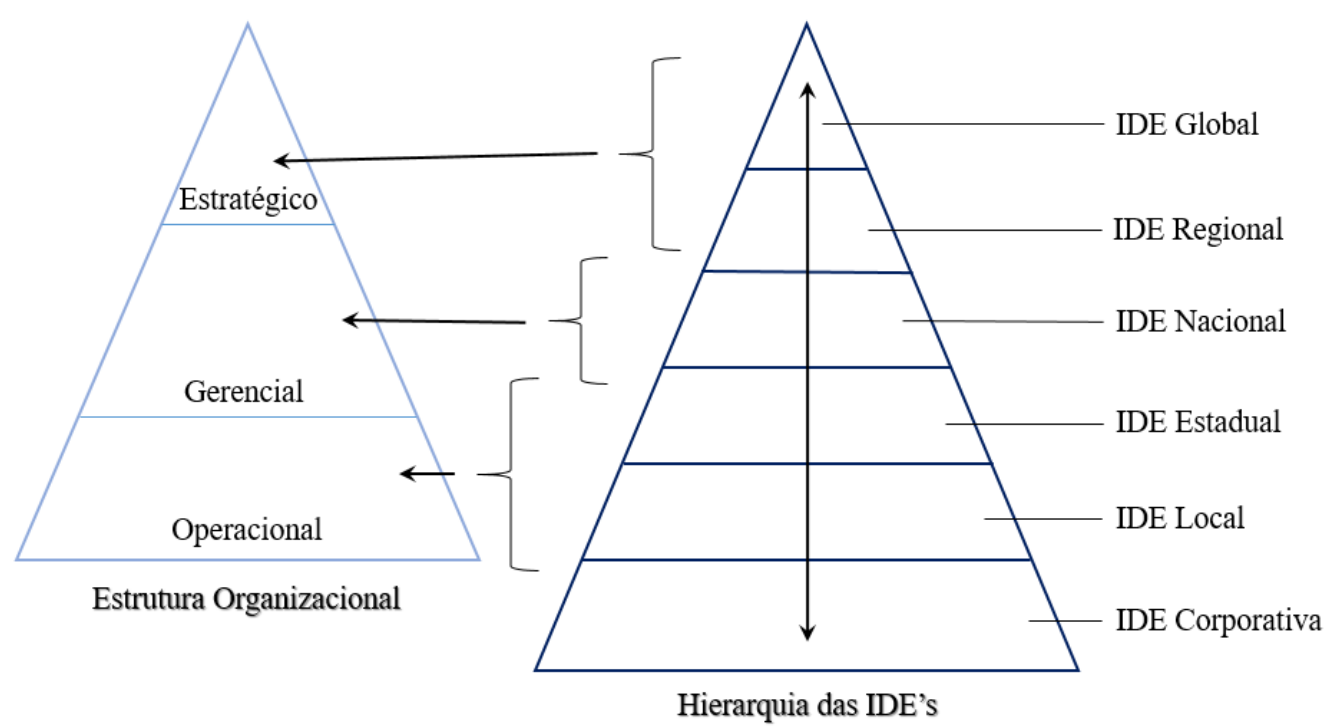

Fonte: Adaptado de Rajabifard, Feeney e Williamson (2002) e CONCAR (2010). 
Nebert (2004) aponta que o aumento do uso de ferramentas interoperáveis de geoserviços, por meio da internet e da capacidade de processamento dos dados espaciais, permitiu a aplicação dos conceitos de gerenciamento de bancos de dados espaciais às IDE's. Cabe ressaltar que a evolução tecnológica combinada ao uso de padrões proporcionou ainda o relacionamento entre as diferentes IDE's, aumentando sua gama de aplicações. Assim, com a chegada da Web 2.0 em 2007, houve o estabelecimento da $3^{\circ}$ geração de IDE, a qual possui centro no usuário (BORBA et al., 2015). Atualmente, a "internet das coisas", "Big Data", "Crowdsourcing", "variáveis dinâmicas" e outros conceitos afins às IDEs têm sido elaborados visando principalmente o armazenamento em nuvem, permitindo maior mobilidade e rapidez ao acesso do usuário (BORBA et al., 2015). A Figura 2, ilustra de forma simplificada os principais componentes de uma IDE contemplando o usuário, conteúdo geográfico, uso de padrões de acesso e intercâmbio, apoiados pelo componente tecnológico de armazenamento e serviços.

Figura 2 - Componentes principais de uma IDE

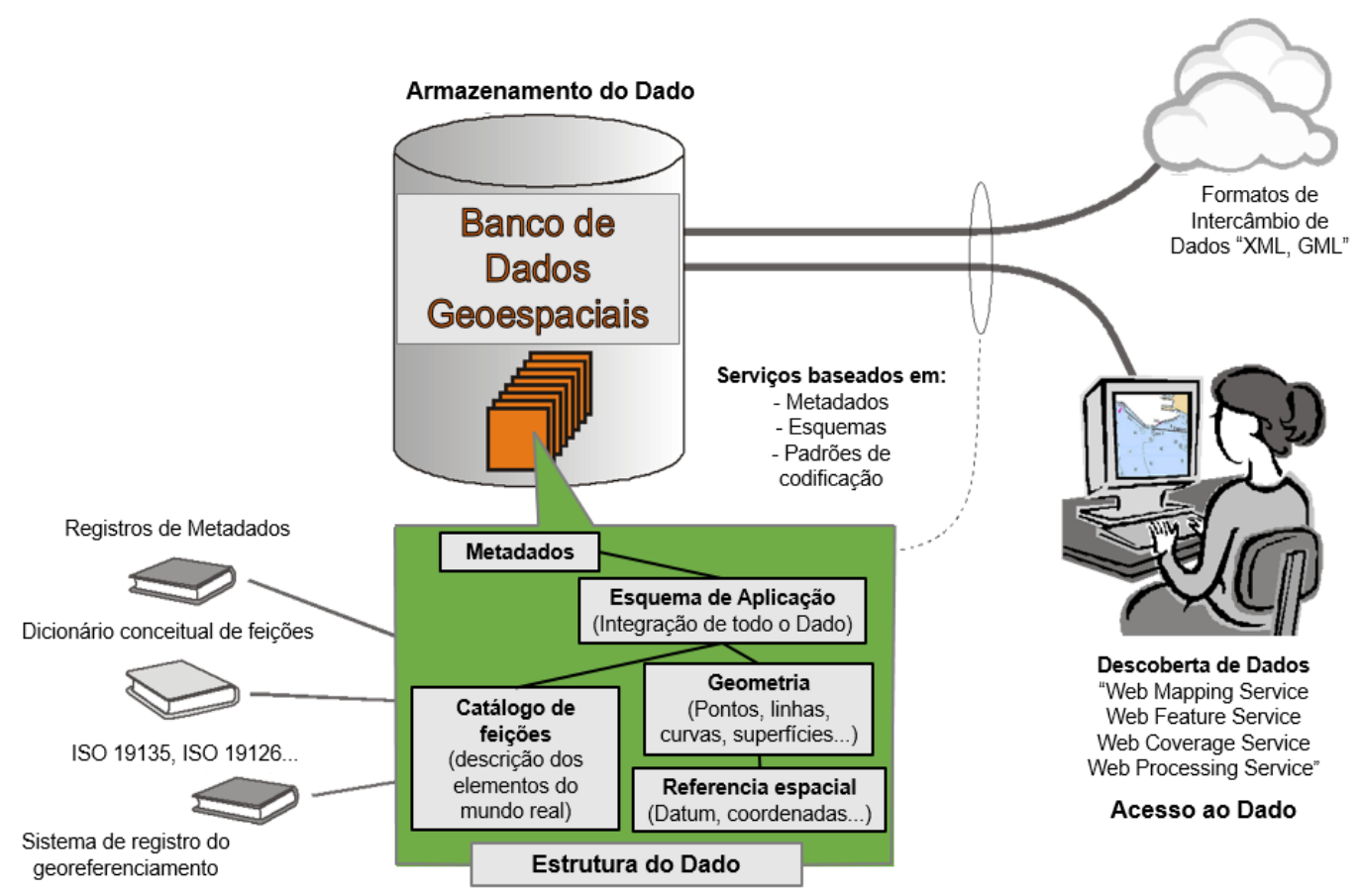

Fonte: Adaptado de Fadaie (2016). 
2.3 A origem das Infraestruturas de Dados Espaciais Marinhos nacionais

Como consequência natural do processo de aperfeiçoamento das IDE's, logo seus limites geográficos alcançaram as regiões marinhas. Responsável por uma cobertura superior à $70 \%$ da superfície terrestre, os mares e oceanos têm sido a maior fonte de alimento e energia à humanidade, mantendo a regulação climática e permitindo a conexão entre as nações para o desenvolvimento mútuo e o comércio (UN-OCEANS, 2016). Desta forma, a busca pelo gerenciamento eficiente de tais recursos trouxe às IDE's à necessidade de integração das informações espaciais por meio do acesso e compartilhamento de dados costeiros e marinhos (STRAIN, RAJABIFARD e WILLIAMSON, 2006) (Figura 3).

Figura 3 - Combinação entre os sistemas de gerenciamento terrestre e marinho

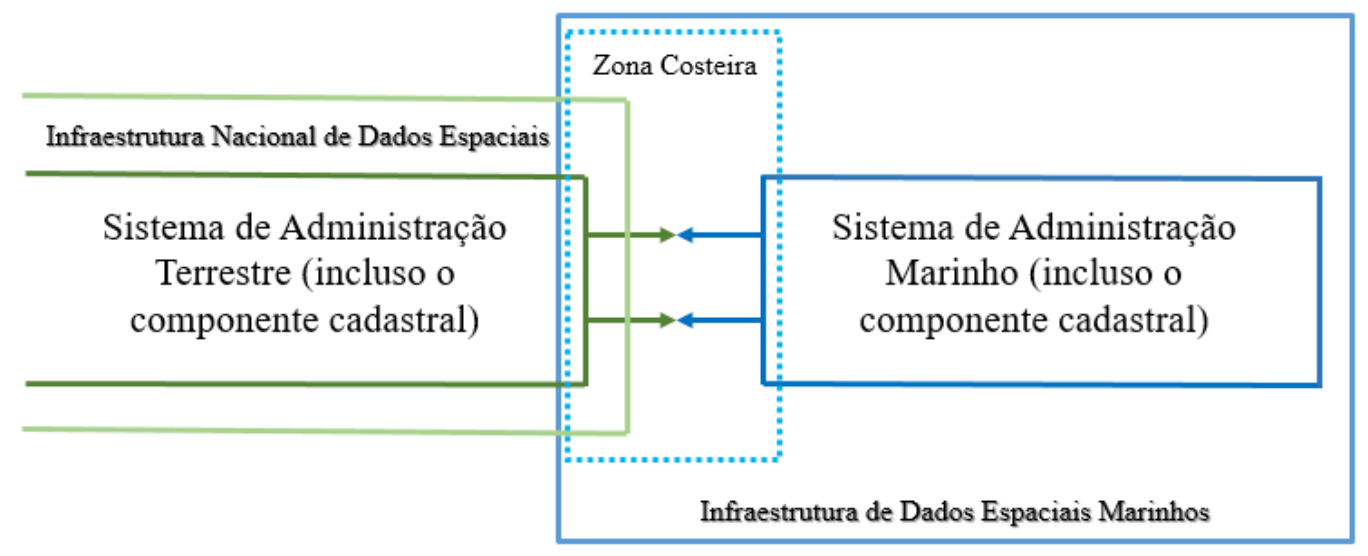

Fonte: Adaptado de Strain, Rajabifard e Williamson (2006).

Ao longo das décadas de 80 e 90, destacaram-se as observações das nações sobre o enorme potencial de riquezas a serem exploradas em seus mares territoriais, zonas contíguas, zonas econômicas exclusivas e plataforma continental, podendo esta ainda ser estendida conforme o atendimento aos critérios estabelecidos pela III Convenção das Nações Unidas sobre os Direitos do Mar (UNCLOS, 1994). Com isso, houve um aumento do interesse mundial a respeito das informações espaciais atinentes ao prolongamento de 
seus territórios para além da linha de costa (sua extensão, limites e recursos naturais), bem como da salvaguarda e da responsabilidade pela gestão integrada destes ambientes.

Assim, conforme Strain, Rajabifard e Williamson (2006), os dados espaciais marinhos que adquiriram maior relevância passaram a constituir as IDEM's, sendo organizados em camadas referentes a fronteiras, áreas de proteção ambiental, rotas de navegação e infraestrutura portuária, climatologia e meteorologia, regime de marés, ondas e correntes, recursos vivos e não vivos, composição do fundo e sub-fundo, além de outras questões legais nacionais e internacionais (Figura 4).

Figura 4 - Exemplo da organização dos temas de uma IDEM

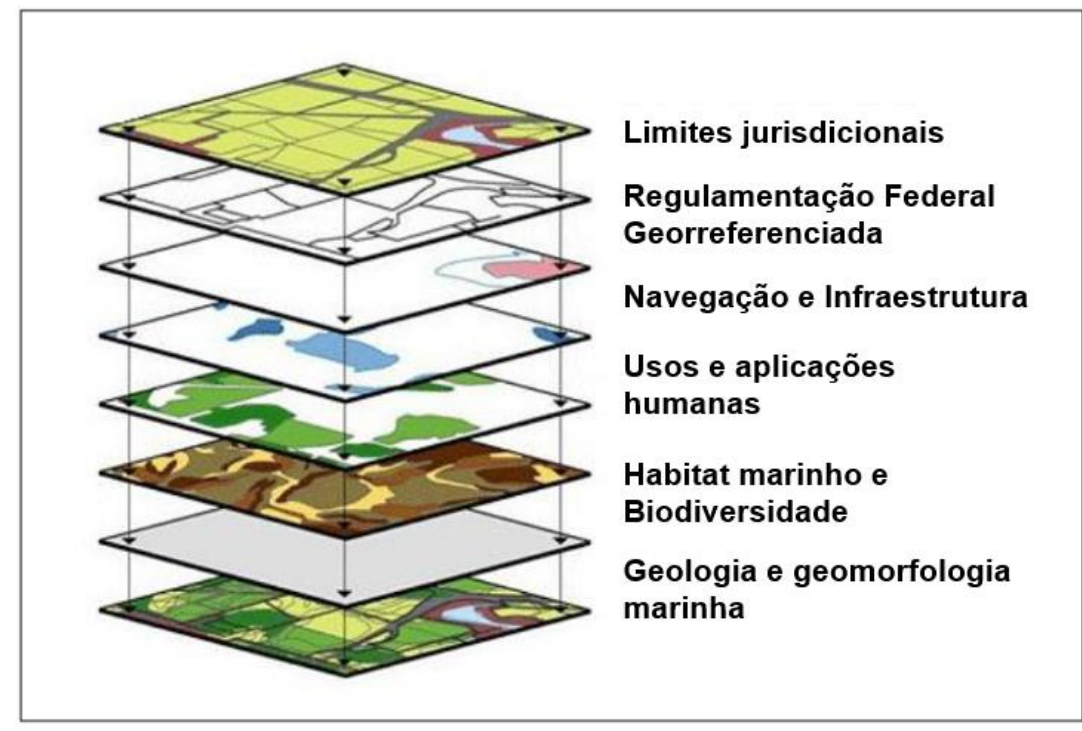

Fonte: Adaptado de Fowler, Smith e Stein (2010).

\subsection{O papel dos Serviços Hidrográficos}

Em meio a esse movimento, Maratos (2006) ressalta a necessidade de preparação dos Serviços Hidrográficos (SH) quanto à aplicação dos dados e informações hidrográficas para além dos propósitos consagrados à segurança da navegação. A crescente relevância do tema também pode ser verificada com a aderência da própria Organização Hidrográfica Internacional (OHI) 
junto ao assunto, sendo esta uma renomada entidade intergovernamental composta por mais de 75 Estados Membros, dentre eles o Brasil (Figura 5). Em seu âmbito, observam-se manifestações com o desempenho de esforços contínuos para apoio de seus partícipes à construção de suas Infraestruturas de Dados Espaciais Marinhos (IDEM). Um exemplo foi o estabelecimento, logo em 2008, do Marine Spatial Data Infrastructures Working Group (MSDIWG) (IHO, 2019a), com intuito de incentivar a troca de experiências e aumentar a eficiência na produção de dados geoespaciais, melhorando assim a disponibilidade e a acessibilidade destes dados e informações.

Figura 5 - Estado membro da OHI (cor azul), Membro suspenso (cor marrom), Estado em processo de incorporação (cor vermelha) e em processo de adesão (cor verde); os demais Estados (cor cinza) não possuem costa ou não manifestaram interesse em participar da OHI

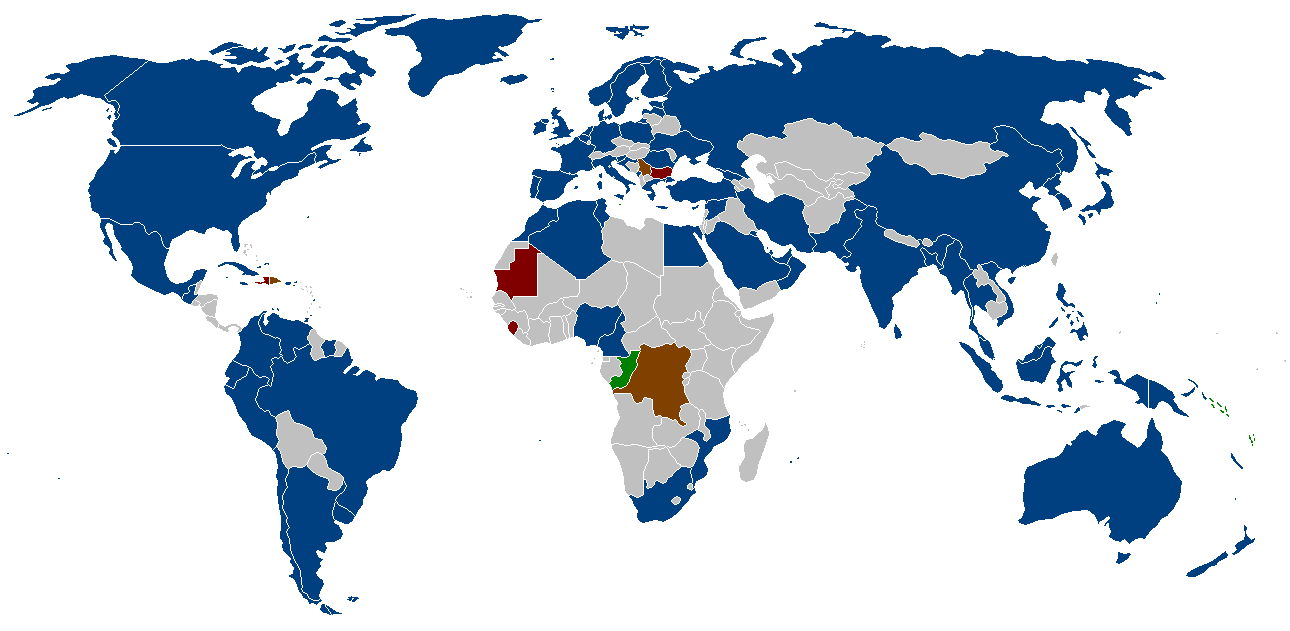

Fonte: IHO (2018b).

Segundo Cooper, Pepper e Osborne (2010), os Serviços Hidrográficos envolvidos no processo de implementação de uma IDEM, serão capazes de gerenciar e assumir o controle "inteligente" sobre seu(s) oceano(s) com o apoio da navegação eletrônica e do planejamento marinho espacial. Desta forma, o atendimento das necessidades de diversos setores públicos e privados, por meio de IDEM tem sido apontado como um importante passo à disseminação da informação marítima e fluvial nacional (CONTI et al., 2013). 
A OHI, por meio de sua publicação C-17 (IHO, 2017a), descreve uma IDEM baseada em quatro pilares, sendo: política e governança (pessoas), especificações técnicas (padrões), sistemas de informação (tecnologias) e conteúdo geográfico (dados espaciais), conforme ilustrado pela Figura 6.

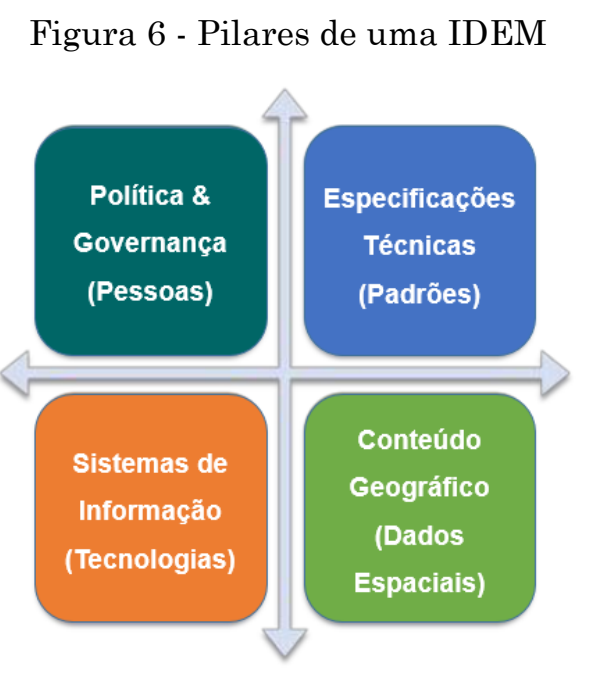

Fonte: Adaptado de IHO (2017a).

Percebe-se que a abordagem das IDEM's no tocante as "Pessoas", difere do modelo de apresentação das IDE's que, geralmente, é baseado em cinco pilares (Pessoas, Dados, Instituições, Tecnologias, Normas e Padrões) (CONCAR, 2010). Neste, as pessoas ou usuários são normalmente representados como um elemento distinto. Já no caso das IDEM's, observa-se que a componente "Políticas e Governança" compreende as ações manifestadas pelas próprias pessoas. Estes atores, que por vezes podem representar uma instituição, detendo autoridade e responsabilidades na gestão dos atos relacionados a IDEM, ocupam o lugar dos gestores e usuários que irão colher os benefícios concedidos pelo incentivo e uso das boas práticas no manuseio das IDEM's. A OHI, busca então difundir uma visão do comprometimento das "Pessoas" com os processos e resultados das IDEM's (HARTMAMM, 2019). Para tal, o IHO-MSDIWG contempla este tópico nas orientações fundamentais dos treinamentos sobre IDEM's (IHO, 2017a). 
Destaca-se também que devido as características oriundas dos dados hidrográficos e aquelas envolvidas nos processos de produção e manutenção dos documentos náuticos, incluso as cartas náuticas, observa-se a adoção pelos SH de "Especificações Técnicas" e formatos abertos padronizados internacionalmente pela OHI. Caso das publicações S-4, INT1/2 para as cartas impressas em papel; bem como da especificação S-57 (IHO Transfer Standard for Digital Hydrographic Data) para os formatos digitais (Electronic Navigational Chart - ENC ou Raster Navigational Chart - RNC).

Ademais, o grande volume de informações e a necessidade de maior interação entre o usuário e seus produtos têm incentivado a busca por "Sistemas de Informação" voltados às especificidades do ambiente marinho (PINHEIRO e FLORENTINO, 2017). Estas tecnologias, sejam elas soluções abertas ou privadas, tendem a colaborar com o atual desenvolvimento do novo Modelo de Dados Hidrográficos Universal “S-100" (IHO, 2018a). Tanto a S-57 quanto a S-100 baseiam-se na série 19.100 do projeto ISO/TC 211 Geographic Information/Geomatics (Comitê Técnico 211 Informação Geográfica/Geomática), da International Organization for Standardization (ISO), visam total conexão aos padrões OGC (Open Geospatial Consortium) e, por consequência, às demais IDE's existentes (FADAIE, 2016).

Por "Dados Espaciais", entende-se o conjunto de elementos: limites marítimos, áreas de conservação e preservação, habitats marinhos, oceanografia, batimetria, hidrografia, geologia, infraestrutura marítima, localização de naufrágios, instalações offshore, tubulações e cabos submarinos etc (IHO, 2017a).

\section{O relacionamento entre as IDE's e IDEM's}

O estabelecimento de uma IDEM ou a adaptação de uma IDE préexistente para agregar os componentes marinhos é uma tendência mundial (IDREES, 2015; IHO, 2019a). Balla e Wouters (2017) apresentam que países como Austrália, Estados Unidos da América e Canadá, atentaram para o 
processo de aperfeiçoamento de suas Infraestruturas de Dados Espaciais (IDE's) terrestres para a temática marinha antes mesmo da iniciativa europeia. Tal fato fez com que diversos países tomassem estes projetos pioneiros como objeto de análise para definição de modelos conceituais de suas próprias Infraestruturas de Dados Espaciais Marinhos (IDEM), caso da Alemanha por exemplo (SEIP e BILL, 2016).

Pode-se ainda citar as infraestruturas: INSPIRE (2018), que serviu de base aos programas específicos de dados marinhos europeu EMODnet (2016; 2018) e SeaDataNet (2018), ou a Malaysia Geospatial Data Infrastructure (MyGDI; 2018) que evoluiu a partir da National Infrastructure for Land Information System (NaLIS). O Land Information New Zealand (LINZ, 2018), tal qual o projeto GeoConnections do Natural Resources Canada (NRCAN, 2018), se destacam ainda pela adoção do modelo de IDEM coerente a iniciativa conhecida por "Open Government". Sendo lançada em setembro de 2011, por um grupo de 8 países (Brasil, Indonésia, México, Noruega, Filipinas, África do Sul, Reino Unido e Estados Unidos da América), e logo endossada voluntariamente por outras 67 nações; a "Parceria de Governo Aberto" declara que os mesmos seguirão uma série de compromissos relacionados a gestão transparente e de participação popular (OGP, 2016). Observa-se que o uso de IDE's e IDEM's no Brasil, tal como instituído pela INDE-BR (BRASIL, 2008) e manifestado pela iniciativa da DHN (DHN, 2016; 2018) segue nesta mesma direção.

Sobre este mesmo prisma, a OHI publicou um documento intitulado "Realising the benefits of Spatial Data Infrastructures in the Hydrographic Community", promovendo as boas práticas na integração dos Serviços Hidrográficos junto as IDE nacionais (IHO, 2017b).

Destaca-se ainda que a implantação das IDEM's segue suportada por diretivas como: United Nations Committee of Experts on Global Geospatial Information Management - Working Group on Marine Geospatial Information (UN-GGIM WGMGI, 2019), Blue Economy and Blue Growth (2018), G8 Open Data Charter (2018), Smart Oceans (2018); International Oceanographic Data 
and Information Exchange (IODE, 2018); e-Navigation (2018); General Bathymetric Chart of the Oceans (GEBCO, 2019). Estas visam desde o estabelecimento de um tráfego marítimo seguro e eficiente; o aperfeiçoamento na gestão da zona costeira; a exploração sustentável e a otimização de recursos marinhos, como a proteção ambiental e o respeito a defesa nacional (COOPER, PEPPER e OSBORNE, 2010).

\subsection{A INDE e a IDEM brasileiras}

O Brasil teve sua Infraestrutura Nacional de Dados Espaciais (INDEBR) estabelecida pelo Decreto n 6.666, em 2008 (BRASIL, 2008). Tal lei prevê a participação dos órgãos governamentais produtores de dados espaciais entre os quais a Diretoria de Hidrografia e Navegação (DHN) - devendo disponibilizar dados e metadados provenientes de seus produtos, de acordo com padrões nacionais de interoperabilidade. Sendo a DHN o Serviço Hidrográfico Nacional, cabe a ela gerir as ações de planejamento e execução da cartografia náutica no Brasil (BRASIL, 1967).

Para melhor coordenação das atividades interinstitucionais, foi estabelecido o Plano de Ação para Implantação da INDE (CONCAR, 2010), que reconhece como dados "geoespaciais especiais" aqueles relacionados à cartografia náutica e desta forma, sob a responsabilidade da DHN. Atualmente, o Ciclo III deste Plano compreende as ações de preparação e implantação das bases de dados no período de 2015 a 2020.

Segundo Körting et al. (2016), uma revisão dos principais tópicos da literatura nacional especializada aponta o surgimento de novas arquiteturas de IDE's como uma das tendências ao universo da geoinformática. Ainda, especificamente no caso da INDE-BR, percebe-se que algumas dificuldades relacionadas a casos de transmissão de grandes volumes de dados espaciais (conjuntos oriundos de sensoriamento remoto ou de levantamentos batimétricos, por exemplo) e o manejo de dados e informações espaciais dinâmicas, ou seja, aquelas com influência temporal (caso das marés ou dados 
climatológicos); têm sido encarados como oportunidades de pesquisa acadêmica e/ou desenvolvimento tecnológico. Trabalhos como de Souto e Menezes (2013) e Conti et. al. (2013) apontavam a necessidade de atenção ao desenvolvimento de parametrizações aos modelos de dados e perfil de metadados, que fossem capazes de representar e absorver futuras informações voltadas ao ambiente marinho. Assim, o desenvolvimento de arquiteturas tal qual às IDEM's mostra-se relevante frente aos desafios de uma gestão transparente, eficiente e sustentável.

Neste sentido, esforços vêm sendo adotados por parte do Serviço Hidrográfico brasileiro, ou seja, pela DHN; com o objetivo de facilitar o acesso e a integração de dados espaciais marinhos por meio da implantação de uma IDEM que venha a atender tanto as demandas apresentadas pelo cenário nacional quanto internacional (DHN, 2016; PINHEIRO e FLORENTINO, 2017).

Conforme recente publicação da Norma de Acesso aos Dados e às Informações Abertas da Diretoria de Hidrografia e Navegação (NAD-DHN) (DHN, 2018), esta infraestrutura de dados espaciais prevê alcançar diversos elementos do ambiente marinho, como os dados de batimetria por exemplo, sejam eles tratados sob a forma discreta ou contínua, caso das superfícies batimétricas interpoladas. Desta forma, observando as normas e padrões nacionais, como as Especificações Técnicas para Estruturação de Dados Geoespaciais Vetoriais "ET-EDGV 3.0" (CONCAR, 2017), bem como o desenvolvimento da modelagem mundial para interoperabilidade de dados marinhos, S-100 (IHO, 2018a), tal IDEM busca promover a descoberta, o acesso e o compartilhamento dos dados e informações espaciais marinhas nacionais de maneira mais eficiente e eficaz, por meio da aplicação de soluções tecnológicas baseadas em bancos de dados espaciais e geoserviços (PINHEIRO e FLORENTINO, 2017). 


\section{Aplicações e Perspectivas Futuras à IDEM Brasileira}

O uso de IDE e IDEM tem alcançado resultados positivos em diversos setores. Como exemplo, as tecnologias geoespaciais representaram benefícios da ordem de $\mathrm{R} \$ 46,067$ bilhões (ou 1,1\%) do produto interno bruto canadense em 2013, sendo os dados espaciais responsáveis pelo acréscimo de $R \$ 1,546$ bilhões a este montante (NRCAN, 2015). Contudo, sabe-se que tais resultados derivam da contínua e efetiva participação das instituições governamentais e privadas que investiram recursos materiais e humanos sobre o projeto da IDEM canadense, que foi uma das primeiras a realizar um estudo de custobenefício para este tema, em 1992 (GRIFFIN, COOTE e CROMPVOETS, 2017).

A Dinamarca, a qual implementou sua IDEM ao longo de 2015 e 2017, apresenta em recente análise feita pela Danish Geodata Agency (IHO, 2019b); o potencial custo benefício ao estabelecimento de uma nova versão para sua atual infraestrutura, a qual será chamada de MSDI 2.0. Neste estudo, percebe-se que investimentos da ordem de $\mathrm{R} \$ 4,2$ milhões, divididos entre as 11 instituições partícipes, ao longo de 2019 a 2022; representariam benefícios anuais líquidos e duradouros a serem compartilhados entre às mesmas, da ordem de $\mathrm{R} \$ 1,2$ milhões a partir de 2024 .

Desta forma, segue uma breve abordagem dos benefícios e das possíveis problemáticas envolvidas sob um cenário de implantação e operação deste projeto a nível nacional.

\subsection{O Potencial dos Dados Espaciais Marinhos}

De forma geral, estudos recentes sobre relações de custo-benefício à implementação de IDEM's nacionais e regionais, afirmam ter encontrado razões positivas variando da ordem de 1:2 alcançando até 1:18, implicando assim em benefícios significativos à estas nações. Normalmente, estes se estendem à aspectos gerais de comércio, a economia de tempo e dinheiro na 
aquisição de novos dados e informações espaciais para suporte a obras e à processos de tomada de decisão, bem como o desenvolvimento de pesquisas marinhas com a otimização de recursos e redução das incertezas (GRIFFIN, COOTE e CROMPVOETS, 2017).

Assim, para efeito da análise sobre um dado espacial oriundo de IDEM's de forma específica, pode-se constatar sua relevância a diversos setores, analisando seus impactos e alcance. A informação batimétrica, por exemplo, é considerada um dado de referência para os demais elementos geográficos, podendo ser representada através de modelos digitais de terreno (MDT) ou mesmo na forma de nuvem de pontos (IHO, 2017a), conforme Figura 7.

Figura7 - Apresentação do dado batimétrico por meio de uma nuvem de pontos (imagem à esquerda) e de um MDT (imagem à direita)
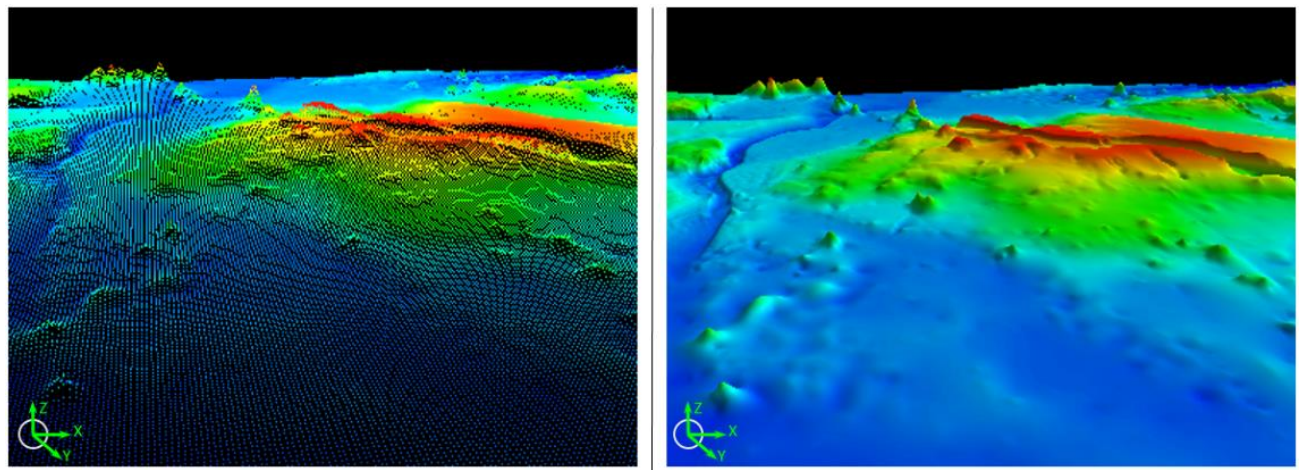

Fonte: Elaborada pelos autores.

Desta forma, percebe-se que este dado geoespacial ultrapassa seu uso tradicional relacionado apenas à segurança da navegação, exercendo influência em aspectos relacionados ao desenvolvimento do contexto nacional, seja em relação ao conhecimento e defesa do território, ao gerenciamento costeiro, ao conhecimento dos recursos marinhos, às relações internacionais de transporte ou ao comércio existente nos dias atuais.

Em se tratando da disponibilização de dados batimétricos de referência, um estudo realizado em 2015, pelo Land Information New Zealand (LINZ) observa detalhadamente os benefícios tanto no campo 
técnico-científico como no desenvolvimento dos principais setores econômicos daquele país. Este estudo aponta que investimentos na coordenação da produção de informações batimétricas nacionais podem apresentar uma relação de custo-benefício da ordem de 1:2 a 1:6. Como exemplo, o Porto de Otago na Nova Zelândia, planeja executar aperfeiçoamentos em sua infraestrutura com base num projeto de dragagem para aumento da profundidade de seus canais para 14 metros (valor compatível às demandas comerciais mundiais). A razão para este desenvolvimento é prover segurança às visitas de navios maiores. Assim, segue estimado que o investimento à época, de $R \$ 167,791$ milhões no Porto de Otago, se traduza em um retorno de mais de $\mathrm{R} \$ 516,280$ milhões nos próximos 20 anos, ou seja da ordem de 1:3. Estes resultados são esperados com base na redução da duplicidade de esforços administrativos e financeiros, priorização na realização dos levantamentos hidrográficos necessários, bem como melhora da disponibilização e da qualidade do dado destinado ao propósito final (LINZ, 2015).

Neste sentido, diversas iniciativas nacionais (LINZ, 2015), regionais (EMODnet, 2016; HALL, 2014) e globais (GEBCO, 2019) buscam atender ao mapeamento do fundo marinho através da aplicação de novas tecnologias de coleta, processamento, análise, controle e compartilhamento de dados batimétricos (IHO, 2017b). Têm-se como exemplos os projetos do IHO Crowdsourced Bathymetry Working Group, iniciado em 2014 (IHO, 2014) e o The Nippon Foundation - GEBCO - Seabed 2030, estabelecido em 2016 (JAKOBSSON et al., 2017; MAYER et al., 2018).

4.1.1 Benefícios esperados com o fornecimento de uma superfície batimétrica pela IDEM brasileira

No Brasil, estudos recentes desenvolvidos pela Diretoria de Hidrografia e Navegação (DHN) em conjunto da Universidade Federal Fluminense (UFF) sobre este quesito têm apontado o potencial uso da superfície batimétrica 
oriunda do Projeto LEPLAC (Levantamento da Plataforma Continental) como referência a iniciativa de implementação da IDEM nacional (FLORENTINO, 2018).

Espera-se que tais dados venham a contribuir em diversas atividades, como nas pesquisas envolvendo a modelagem numérica e a geomorfologia marinha. No âmbito da modelagem meteorológica e/ou oceanográfica, a melhora na qualidade dos dados de contorno, ou seja, da superfície batimétrica, pode influenciar positivamente nas previsões meteoceanográficas (GABIOUX et al., 2013; LACASCE, 2017), representando um ganho à segurança da navegação nacional. Já os trabalhos envolvendo a definição e estudo de feições geomorfológicas marinhas apontariam o aumento do domínio e compreensão da geomorfologia local e, consequentemente, da definição do pé do talude e da isobatimétrica de $2.500 \mathrm{~m}$, elementos determinantes para as análises em curso pela Comissão de Limites da Plataforma Continental (CLPC) para extensão dos limites territoriais nacionais de acordo com Art. $n^{\circ} 76$ da UNCLOS (MOHRIAK e TORRES, 2017). Ademais, o avanço em pesquisas para caracterização geomorfométrica pode subsidiar modelos de previsão de movimentos de massa submarinos mais acurados atuando assim sobre possíveis desastres naturais que afetariam estruturas de produção de óleo e gás offshore (CHIOCCI, CATTANEO e URGELES, 2011; LECOURS et al., 2016).

O fornecimento deste dado batimétrico atenderia aos anseios do Plano de Ação para Implantação da INDE-BR (CONCAR, 2010), e demonstraria o elevado grau de compromisso das instituições responsáveis na adoção de políticas de transparência e sustentabilidade junto a sociedade brasileira.

Por fim, tal como apresentado pela iniciativa do projeto EMODnet Bathymetry (2016), a adoção e fornecimento de uma superfície batimétrica (nacional ou regional) rica em dados batimétricos devidamente analisados e qualificados, pode vir a subsidiar a melhora de modelos globais como a própria superfície GEBCO_2019. Esta ação é fortemente apoiada por renomadas 
instituições internacionais, como a OHI, a GEBCO e a COI (Comissão Oceanográfica Intergovernamental) (IHO e IOC, 2018).

\subsection{Desafios à implementação e operação de uma IDEM}

Ainda que os benefícios de tal iniciativa sejam na maioria dos casos superiores a razão de 1:2 alçando até 1:18 (GRIFFIN, COOTE e CROMPVOETS, 2017), faz-se necessário firmar o compromisso adequado com o provimento de recursos materiais e humanos para a garantia do sucesso. Assim, a implementação de uma IDEM eficiente implica na superação de vários obstáculos.

Existem custos diretos e indiretos associados a uma iniciativa dessa magnitude (JOHNSON et al., 2017) e um passo à revelia de um planejamento adequado pode implicar na percepção rasa de que sua implementação consiste em algo extremamente simples, fácil e de nenhum custo. Contudo, Griffin, Coote e Crompvoets (2017) descrevem em um estudo comparativo sobre as relações de custo-benefício de oito relevantes IDEM's nacionais e regionais em operação, que os principais custos mensuráveis se devem aos esforços de levantamento dos dados espaciais marinhos, sua estrutura de gerenciamento e análise.

Aspectos envolvendo a coleta e atualização dos dados, além de sua respectiva preparação para uma plataforma aberta, muitas vezes on-line, demandam a dedicação e reciclagem contínua de pessoal técnico qualificado e de tempo (FADAIE, 2016). Os dados também devem ser preparados para o intercâmbio entre as diversas agências de governo ou privadas que alimentam estas estruturas, implicando em um dispêndio de tempo e recursos que muitas vezes não se traduz num retorno notório direto para os fornecedores (IDREES, 2015).

Soma-se a necessidade do desenvolvimento de mecanismos dedicados a compatibilização dos modelos conceituais marinhos e terrestres, inclusive contemplando a expansão do perfil de metadados e seus padrões de 
intercâmbio (SOUTO e MENEZES, 2013; CONCAR, 2017; IHO, 2018a). Além do desempenho requerido ao manuseio de significativo volume de dados e atualizações constantes, desafios em termos de visualização e aproveitamento destas informações de modo intuitivo ao usuário são questionamentos impostos aos Serviços Hidrográficos para o desenvolvimento científico e tecnológico das IDEM's (HARTMANN, 2015; IHO, 2019a).

Assim, à luz das regulamentações vigentes, deve haver ainda uma filtragem e triagem, de modo a proteger dados confidenciais (sejam eles pessoais, econômicos ou militares), garantindo que direitos autorais não venham a ser violados (CONCAR, 2010). Além da busca por assegurar a qualidade dos serviços prestados por todo esse conjunto, devem ser realizados investimentos envolvendo treinamento de pessoal, desenvolvimento e aprimoramento de normas e regras, bem como no preparo de pessoas para lidar com o feedback dos usuários (JOHNSON et al., 2017).

\section{Conclusão}

Como parte das modernas IDE's, o desenvolvimento das IDEM's tem ocorrido como consequência direta à busca por soluções ao gerenciamento eficiente e sustentável das atividades humanas nas escalas nacional, regional e global, que sejam integradas e de cunho estratégico.

Uma análise sobre a evolução das iniciativas já em operação por alguns países demonstra os inúmeros benefícios de sua implantação aos diversos setores da sociedade. Seja no cenário terrestre ou marinho, diretivas nacionais e internacionais reforçam a relevância da correta catalogação, armazenamento e compartilhamento destes dados espaciais tão valiosos às atividades atuais e futuras.

O estudo mais aprofundado sobre o papel de um tipo de dado espacial marinho, como o exemplo descrito da batimetria, demonstra o potencial alcance da influência do mesmo frente a uma relação de custo-benefício. Contudo, ressalta-se que para a real e eficiente operação deste modelo, faz-se 
necessário o aporte de investimentos desde a capacitação de pessoal e aquisição de material técnico, como para a implementação e garantia da manutenção dos serviços atinentes a esta estrutura.

Portanto, representando um relacionamento complementar entre as ações propostas pela INDE-BR, o desenvolvimento harmônico de uma IDEMBR mostra-se oportuno, pois age também como um dispositivo de conexão internacional. Este demonstra reforçar os valores nacionais de transparência e participação do Estado junto à sociedade em prol do bem comum.

\section{Contribuição dos autores}

O autor Christopher Florentino contribuiu em todo o processo, desde a concepção do artigo, sua estruturação metodológica, escrita e análise conceitual. Vitor Bravo Pimentel contribuiu com a discussão e revisão do texto. Arthur Ayres Neto foi responsável pela orientação conceitual e revisão do manuscrito.

\section{Referências}

AALDERS, H. J. G. L.; MOELLERING, H. Spatial Data Infrastructure. Proceedings of the 20th International Cartographic Conference. Beijin, 2001. Site <https://icaci.org/files/documents/ICC_proceedings/ ICC2001/icc2001/file/f14005.pdf>. Acessado em julho de 2018.

BALLA, E.; WOUTERS, R. Marine Cadastre in Europe: State of Play. 2017 World Bank Conference on Land and Poverty. 2017. Site <https://www.iho.int/ mtg_docs/com_wg/MSDIWG/MSDIWG9/MSDIWG9-07F2-Marine_Cadastre_ Europe.pdf>. Acessado em abril de 2019.

BORBA, R. L. R.; STRAUCH, J. C. M.; SOUZA, J. M. DE; COLEMAN, D. J. Uma Proposta para a Nova Geração de Infraestrutura de Dados Espaciais. Revista Brasileira de Cartografia, v. 67, n.6, p. 1145-1166, 2015. 
BRASIL. Decreto-Lei no 243, de 28 de fevereiro de 1967. Fixa as Diretrizes e Bases da Cartografia Brasileira e dá outras providências. Diário Oficial da União, Seção 1, p. 2438, 28 de fevereiro de 1967.

BRASIL. Decreto $n^{0}$ 6666, de 27 de novembro de 2008. Institui, no âmbito do Poder Executivo Federal, a Infraestrutura Nacional de Dados Espaciais - INDE, e dá outras providências. Brasília, Diário Oficial da União, 27 de novembro de 2008 .

BLUE ECONOMY AND BLUE GROWTH. Site <https://ec.europa.eu/ maritimeaffairs/policy/blue_growth_en>. Acessado em outubro de 2018.

CHEN, J. Singapore's National Marine Spatial Data Infrastructure (NMSDI) Concept: GeoSpace-Sea. 2019. Site <https://portal.opengeospatial.org/ files/?artifact_id=83030>. Acessado em abril 2019.

CHIOCCI, F. L.; CATTANEO, A.; URGELES, R. Seafloor mapping for geohazard assessment: State of the art. Marine Geophysical Research, v. 32, n. 1, p. 1-11, 2011.

CONCAR, Comissão Nacional De Cartografia. Plano de Ação para Implantação da Infraestrutura Nacional de Dados Espaciais. 2010. Rio de Janeiro. Site $<$ http://www.concar.gov.br/pdf/PlanoDeAcaoINDE.pdf>. Acessado em agosto de 2018.

CONCAR, Comissão Nacional De Cartografia. Especificações Técnicas para Estruturação de Dados Geoespaciais Vetoriais (ET-EDGV 3.0). 2017. Site <http://www.geoportal.eb.mil.br/portal/inde2?id=139>. Acessado em abril de 2019.

CONTI, L. A.; OliveirA, M. C. de; ESTRADA, T. E. M. D.; MARQUES, A. C. Gerenciamento de dados marinhos no contexto brasileiro. Biota Neotropical, v. 13, n. 2, p. 21-26, 2013.

COOPER, P.; PEPPER, J.; OSBORNE, M. White Paper - The Hydrographic and Oceanographic Dimension to Marine Spatial Data Infrastructure Development: Developing the capacity. 2010. Site <https://www.iho.int/ mtg_docs/com_wg/MSDIWG/MSDIWG_Misc/MSDIWG-BOK.html>. Acessado em agosto de 2018 .

CROMPVOETS, J.; BREGT, A.; RAJABIFARD, A.; WILLIAMSON, I. P. Assessing the worldwide developments of national spatial data clearinghouses. 
International Journal of Geographical Information Science, v. 18, n. 7, p. 665-689, 2004.

DHN, Diretoria de Hidrografia e Navegação. MSDI-WG National Report (BRAZIL). Tóquio, Japão. IHO-MSDIWG 2016. Site < https://www.iho.int/mtg_docs/ com_wg/MSDIWG/MSDIWG7/MSDIWG7-1.6A-National_Report-Brazil.pdf>. Acessado em setembro de 2018.

DHN, Diretoria de Hidrografia e Navegação. Portaria n 13, de 19 de fevereiro de 2018, da DHN. Aprova a Norma de Acesso aos Dados e às Informações Abertas da Diretoria de Hidrografia e Navegação (NAD-DHN). 2018. Site <https://www.marinha.mil.br/dhn/?q=pt-br/nad>. Acessado em agosto de 2018 .

EMODnet, European Marine Observation and Data network. Geoportal. 2018. Site $<$ http://www.emodnet.eu/>. Acessado em setembro de 2018.

EMODnet, European Marine Observation and Data network. EMODnet Digital Bathymetry (DTM), 2016. Site <http://sextant.ifremer.fr/record/c7b53704999d-4721-b1a3-04ec60c87238/>. Acessado em agosto de 2018.

E-NAVIGATION. Site <http://www.iala-aism.org/products-projects/e-navigation/>. Acessado em outubro 2018.

EUROGI, European Umbrella Organisation for Geographic Information. Site $<$ http://eurogi.org/about-us/>. Acesso em setembro de 2018.

FADAIE, K. A comparative study of the development of Marine Spatial Data Infrastructure (MSDI) by IHO Member Nations. Tóquio, Japão. IHOMSDIWG 2016. Site <https://www.iho.int/mtg_docs/com_wg/MSDIWG/ MSDIWG7/MSDIWG7.html>. Acessado em agosto de 2018.

FAPESP, Fundação de Amparo à Pesquisa do Estado de São Paulo. Falta de uma infraestrutura de dados espaciais limita pesquisa oceanográfica no Brasil, diz especialista. 2012. Site <http://agencia.fapesp.br/print/ falta_de_uma_infraestrutura_de_dados_espaciais_limita_pesquisa_oceanogra fica_no_brasil_diz_especialista/15472/>. Acessado em agosto de 2018.

FGDC, Federal Geographic Data Committee. A strategy for the national spatial data infrastructure. 1997. 20p. Site <www.fgdc.gov>. Acessado em agosto de 2018. 
FLORENTINO, C. Análise da qualidade de superfícies batimétricas de referência para uso em Infraestrutura de Dados Espaciais Marinhos. 2018. Dissertação (Mestrado). Universidade Federal Fluminense, Programa de Pós-Graduação em Dinâmica dos Oceanos e da Terra, Rio de Janeiro, 2018. $100 \mathrm{p}$.

FOWLER, C.; SMITH, B.; STEIN, D. Building a Marine Spatial Data Infrastructure to support Marine Spatial Planning in US waters. Geographic Technologies Applied to Marine Spatial Planning and Integrated Coastal Zone Management, n. 1, p. 46, 2010.

GABIOUX, M; COSTA, V. S.; DE SOUZA, J.M.A.C.; OLIVEIRA, B. F.; PAIVA, A. M. Modeling the south atlantic ocean from medium to high-resolution. Revista Brasileira de Geofisica, v. 31, n. 2, p. 229-242, 2013.

G8 OPEN DATA CHARTER. Site <https://www.gov.uk/government/publications/ open-data-charter/g8-open-data-charter-and-technical-annex>. Acessado em outubro de 2018.

GEBCO, General Bathymetric Chart of the Oceans. Site <https://www.gebco.net/>. Acessado em abril de 2019.

GRIFFIN, E.; COOTE, A.; CROMPVOETS, J. A marine spatial data infrastructure in New Zealand: a systematic review on the cost-benefits. Journal of Spatial Science, 15p. 2017. DOI: 10.1080/14498596.2017.1372227.

HALL, J. K. GEBCO Red Sea Grid Underway. Hydro International, p. 6, 2014. Site <http://member.hydro-international.com/articles/item/51b933836ab94d3 db08a4367b8fc83e8>. Acessado em agosto de 2018.

HARTMANN, J. P. W. National Approach in Data Exchange - Danish Maritime Spatial Data Infrastructure. 2nd Baltic Forum of MSP. 2015. Site $<$ http://www.balticscope.eu/content/uploads/2015/07/W2_7_presentation_3_Je ns_Peter_W.Hartmann..pdf>. Acessado em abril de 2019.

IDREES, M. O. Challenges in Coastal Spatial Data Infrastructure implementation: A review. South African Journal of Geomatics. V. 4, n. 4, p. 369-383, 2015. Site <http://www.ajol.info/index.php/sajg/article/view/125781>. Acessado em abril de 2019.

IHO, International Hydrographic Organization. IHO Encourages Crowdsourced Bathymetry. Hydro International. Vol. October, 2014. Site 
$<$ https://www.hydro-international.com/content/article/iho-encouragingcrowdsourced-bathymetry>. Acessado em agosto de 2018.

IHO, International Hydrographic Organization. Spatial Data Infrastructures "The Marine Dimension”. Guidance for Hydrographic Offices (C-17). $n^{\circ} 2.0, p$. $1-45,2017$ a.

IHO, International Hydrographic Organization. White Paper - Realising the benefits of Spatial Data Infrastructures in the Hydrographic Community. 2017b. Site <https://www.iho.int/mtg_docs/com_wg/MSDIWG/ MSDIWG_Misc/MSDIWG-BOK.html>. Acessado em agosto de 2018.

IHO, International Hydrographic Organization. IHO-MSDIWG: Marine Spatial Data Infrastructure Working Group. 2019. Site <https://www.iho.int/ srv1/index.php?option=com_content\&view=article\&id=483\&Itemid=370\&lang $=$ en $>$. Acessado em abril de 2019a.

IHO, International Hydrographic Organization. IHO-MSDIWG. Business Case for a Further Development of the Danish Marine Spatial Data Infrastructure (MSDI). 2019. Site <https://www.iho.int/ mtg_docs/com_wg/MSDIWG/MSDIWG10/MSDIWG10-06A-National_Report-

Denmark.pdf $>$. Acessado em abril 2019b.

IHO, International Hydrographic Organization. S-100 Universal Hydrographic Data Model. IHO Standard S-100. Edition 4.0.0, p. 1-745, 2018a.

IHO, International Hydrographic Organization. IHO Membership. Site $<$ https://www.iho.int>. Acessado em agosto de 2018b.

IHO, International Hydrographic Organization; IOC, Intergovernmental Oceanographic Comission. The IHO-IOC GEBCO Cook Book. 2018. IHO Publication B-11 \& IOC Manuals and Guides n 63.

INSPIRE, Infrastructure for Spatial Information in the European Community. Geoportal. Site <http://inspire.jrc.ec.europa.eu>. Acessado em outubro de 2018.

IODE, International Oceanographic Data and Information Exchange. Ocean Data Portal project. Site <http://www.oceandataportal.org/>. Acessado em outubro de 2018.

JAKOBSSON, M.; ALLEN, G.; CARBOTTE, S.; FALCONER, R.; FERRINI, V.; MARKS, K.; MAYER, L.; ROVERE, M.; SCHMITT, T.; WEATHERALL, P.; 
WIGLEY, R. The Nippon Foundation - GEBCO - Seabed 2030: Roadmap for Future Ocean Floor Mapping. The NIPPON Foundation - GEBCO IHO, UNESCO \& $\quad$ IOC. p.44. 2017. Site $<$ https://www.gebco.net/about_us/seabed2030_project/>. Acessado em agosto de 2018.

JOHNSON, P. A.; SIEBER, R.; SCASSA, T.; STEPHENS, M.; ROBINSON, P. The Cost(s) of Geospatial Open Data. Transactions in GIS, v. 21, n. 3, p. 434-445, 2017.

KÖRTING, T. S.; FERREIRA, K. R.; VINHAS, L.; MIGUEL, A.; MONTEIRO, V.; QUEIROZ, G. R. de. Trends in Geoinformatics. Revista Brasileira de Cartografia, v. 68, Special Issue GEOINFO2015, p.1079-1086, 2016

LACASCE, J. H. The Prevalence of Oceanic Surface Modes. Geophysical Research Letters, p. 1-9, 2017.

LECOURS, V.; DOLAN, M. F. J.; MICALLEF, A.; LUCIEER, V. L. A review of marine geomorphometry, the quantitative study of the seafloor. Hydrology and Earth System Sciences, v. 20, n. 8, p. 3207-3244, 2016.

LINZ, Land and Information New Zealand. New Zealand Bathymetry Investigation. Octuber, 2015. p. 1-83. Site <http://www.linz.govt.nz/aboutlinz/what-were-doing/projects/new-zealand-bathymetry-investigation>. Acessado em agosto de 2018.

LINZ, Land and Information New Zealand. Geoportal. Site $<$ https://www.linz.govt.nz/data/linz-data/hydrographic-data>. Acessado em outubro de 2018.

MyGDI, Malaysia Geospatial Data Infrastructure. Geoportal. Site<http://www.mygeoportal.gov.my/node/3159/>. Acessado em outubro de 2018.

MARATOS, A. The Role of Hydrographic Services with Regard to Geospatial Data and Planning Infrastructure. International Hydrographic Review, v. 7, n. 2, p. 51-53, 2006.

MAYER, L.; JAKOBSSON, M.; ALLEN, G.; DORSCHEL, B.; FALCONER, R.; FERRINI, V.; LAMARCHE, G.; SNAITH, H.; WEATHERALL, P. The Nippon Foundation-GEBCO Seabed 2030 Project: The Quest to See the World's 
Oceans Completely Mapped by 2030. Geosciences, v. 8, n. 2, p. 63, 2018. Site <http://www.mdpi.com/2076-3263/8/2/63>. Acessado em junho de 2018.

MCLAUGHLIN, J. Towards National Spatial Data Infrastructure. In Proceedings of the 1991 Canadian Conference on GIS. 1991. Ottawa, Canada.

MDI-DE - Marine Dateninfrastruktur Deutschland. Geoportal. Site <https:// www.mdi-de.org>. Acessado em junho de 2018.

MOHRIAK, W. U.; TORRES, L. C. Levantamentos geofísicos para a delimitação da margem continental brasileira. Revista USP, v. Abril/Maio, n. 113, p. 59-80, 2017.

NEBERT, D. D. Developing Spatial Data Infrastructure: The SDI Cookbook. GSDI Cookbook. $2^{\circ}$ ed. 2004. Site <www.gsdi.org>. Acessado em agosto de 2018.

NRCAN, Ministery of Natural Resources Canada. Canadian Geomatics Environmental Scan and Value Study. 2015. Site $<$ http://geoscan.nrcan.gc.ca/starweb/geoscan/servlet.starweb?path=geoscan/ful le.web\&search1=R=296426>. Acessado em agosto de 2018.

NRCAN, Ministery of Natural Resources Canada. GeoConnections. 2018. Site <http://www.nrcan.gc.ca/earth-sciences/resources/tools-applications/ 10790>. Acessado em outubro de 2018.

OGC, Open Geospatial Consortium. OGC History (detailed). 2018. Site $<$ http://www.opengeospatial.org/ogc/historylong $>$. Acessado em setembro de 2018.

OGP, Open Government Partnership. Open Government Declaration. OGP Brochure, 2016. Site <http://www.opengovpartnership.org/sites/ default/files/attachments/leaflet_web.pdf $>$. Acessado em outubro de 2018.

PINHEIRO, J. G.; FLORENTINO, C. Infraestrutura de dados espaciais marinhos: uma aplicação na cartografia, hidrografia e oceanografia. Anais do XXVII Congresso Brasileiro de Cartografia e XXVI Exposicarta. Rio de Janeiro: Sociedade Brasileira de Cartografia, Geodésia, Fotogrametria e Sensoriamento Remoto, 2017. Site <http://www.cartografia.org.br/cbc/2017/trabalhos/6/ fullpaper/CT06-17_1506717034.pdf>. Acessado em junho de 2018. 
RAJABIFARD, A. A Spatial Data Infrastructure for a Spatially Enables Government and Society. In: A Multi-View Framework to Assess Spatial Data Infrastructures. Chapter 1, p. 11-22, 2008.

RAJABIFARD, A.; FEENEY, M.; WILLIAMSON, I. P. Directions for the Future of SDI Development. International Journal of Applied Earth Observation and Geoinformation, v. 4, p. 11-22, 2002. Site $<$ http://www.csdila.ie.unimelb.edu.au/publication/journals/Future_Directionsf or_of_SDI_Development.pdf $>$. Acessado em agosto de 2018.

SEADATANET. Site < https://www.seadatanet.org/>. Acessado em outubro de 2018.

SEIP, C.; BILL, R. A Framework for the Evaluation of Marine Spatial Data Infrastructures - Accompanied by International Case-Studies. GeoScience Engineering, v. 62, n. 2, p. 27-43, 2016. Site <https://doi.org/10.1515/gse2016-0015>. Acessado em abril de 2019.

SMART OCEANS. Site <http://www.globalopportunitynetwork.org/report-2016/ smart-ocean/\#.Vx3SrnrHmv8>. Acessado em outubro de 2018.

SOUTO, R. D.; MENEZES, P. M. L. de. A representação dos ambientes costeiro e marinho nas infraestruturas de dados espaciais para fins de gerenciamento costeiro. Revista Brasileira de Cartografia, v. 65, n. 6, p. 1087-1096, 2013.

STRAIN, L. M.; RAJABIFARD, A.; WILLIAMSON, I. Marine administration and spatial data infrastructure. Marine Policy, v. 30, n. 4, p. 431-441, 2006.

TAVRA, M.; LEDER, T. D.; CETL, V. Stakeholders Needs Requisite Analysis: Towards Croatian Marine Spatial Data Infrastructure Establishment. Tehnicki vjesnik - Technical Gazette. v. 25, p. 176-182, 2018. Site https://doi.org/10.17559/TV-20160607222834>. Acessado em abril 2019.

UN-GGIM WGMGI, United Nations Committee of Experts on Global Geospatial Information Management - Working Group on Marine Geospatial Information. Summary Report of First Expert Meeting of the WGMGI. Busan, Coréia do Sul, março de 2019. Site <http://ggim.un.org/meetings/2019/WG-MGIBusan>. Acessado em abril de 2019.

UN-OCEANS. UN Atlas of the Oceans: Facts. 2016. Site $<$ http://www.oceansatlas.org/facts/en>. Acessado em agosto de 2018.

UNCLOS, United Nations Division for Ocean Affairs and Law of the Sea. 3rd United Nations Convention on the Law of the Sea. 1994, p. 7-208. 